\title{
Efeitos da Suplementação de Probióticos e Simbióticos na Doença Hepática Gordurosa Não Alcoólica: Revisão Sistemática
}

\author{
Letícia Cristinne Costa da Silva, ${ }^{1}$ Luiza Marly Freitas de Carvalho ${ }^{2}$
}

\begin{abstract}
RESUMO
Objetivo: Realizar uma revisão sistemática acerca das evidências científicas sobre os efeitos da suplementação de probióticos e simbióticos em portadores da doença hepática gordurosa não alcoólica. Método: Trata-se de um estudo de revisão sistemática realizado nas bases de dados MedLine via PubMed, Web of Science e Scopus, no mês de junho de 2020, utilizando os descritores: non-alcoholic fatty liver disease, probiotics, synbiotic. Após aplicação dos critérios de elegibilidade, permaneceram 12 ensaios clínicos intervencionais. Resultados: A suplementação de probióticos e simbióticos tem apresentado resultados promissores na doença hepática gordurosa não alcoólica. A maioria dos ensaios clínicos analisados demonstrou redução da concentração sérica das enzimas hepáticas, melhora do perfil lipídico e diminuição do grau de esteatose hepática após a intervenção. As cepas probióticas utilizadas em todos os estudos foram dos gêneros Lactobacillus e Bifidobacterium. Os prebióticos mais usados na composição dos simbióticos foram os frutooligossacarídeos e inulina. Conclusão: Ainda há poucos ensaios clínicos publicados e os estudos foram realizados com diferentes prebióticos e espécies de cepas probióticas, dose e tempo de intervenção, portanto não há uma indicação específica para a suplementação. Sugere-se realização de mais estudos com a finalidade de esclarecer a efetividade dos probióticos e simbióticos na doença hepática gordurosa não alcoólica.

Palavras-chave: Doença hepática. Hepatopatia gordurosa não alcoólica. Esteatose hepática. Fígado gorduroso. Disbiose. Microbiota gastrointestinal.

EFFECTS OF PROBIOTIC AND SYMBIOTIC SUPPLEMENTATION ON NONALCOHOLIC FATTY LIVER DISEASE: SYSTEMATIC REVIEW

\section{ABSTRACT}

Objective: To conduct a systematic review on scientific evidence on the effects of probiotic and symbiotic supplementation in patients with non-alcoholic fatty liver disease. Method: This is a systematic review study conducted in Medline databases via PubMed, Web of Science and Scopus, in June 2020, using the descriptors: non-alcoholic fatty liver disease, probiotics, symbiotic. After applying the eligibility criteria, 12 interventional clinical trials remained. Results: Probiotic and symbiotic supplementation has shown promising results in non-alcoholic fatty liver disease, most clinical trials analyzed demonstrated a reduction in serum concentration of liver enzymes, improved lipid profile and decreased degree of hepatic strain after intervention. The probiotic strains used in all studies were of the genus Lactobacillus and genus Bifidobacterium. The prebiotics most used in the composition of symbiotics were fructooligosaccharides and inulin. Conclusion: There are still few published clinical trials and studies have been conducted with different prebiotics and species of probiotic strains, dose and intervention time, so there is no specific indication for supplementation. Further studies are suggested to clarify the effectiveness of probiotics and symbiotics in non-alcoholic fatty liver disease.
\end{abstract}

Keywords: Liver disease. Non-alcoholic fatty liver disease. Hepatic steatosis. Fatty Liver. Dysbiosis. Gastrointestinal Microbiome.

RECEBIDO EM: 11/8/2020

MODIFICAÇÕES SOLICITADAS EM: $14 / 9 / 2020$

ACEITO EM: $14 / 10 / 2020$

\footnotetext{
Autora correspondente. Centro Universitário Santo Agostinho - Unifsa. Av. Prof. Valter Alencar, 665 - 64019-625, São Pedro, Teresina/PI, Brasil. http://lattes. cnpq.br/0960070349313027. https://orcid.org/0000-0003-4099-5201. leticiaccsnutri@gmail.com
}

2 Centro Universitário Santo Agostinho - UNIFSA. Teresina/PI, Brasil. 


\section{INTRODUÇÃO}

O fígado é a maior glândula do corpo humano. Possui atividade endócrina e exócrina e executa mais de 150 funções no organismo, entre elas: atua no metabolismo glicolipídico; manutenção da osmolaridade plasmática; produz proteínas e enzimas essenciais; realiza o catabolismo de tóxicos endógenos; armazena glicogênio, vitamina B12, ferro e cobre; contribui no funcionamento do sistema imunológico (CICERO; COLLETTI; BELLENTANI, 2018).

Nesse contexto, a integridade do fígado, bem como a sua funcionalidade, é essencial para a atividade metabólica adequada do organismo. Uma doença que acometa este órgão pode progredir de aguda à crônica. As principais doenças que cursam para cronificação são as hepatites virais: hepatite $C$ e hepatite B, doença hepática alcoólica e a Doença Hepática Gordurosa Não Alcoólica (DHGNA). Atualmente, a DHGNA vem se tornando uma importante causa de morbimortalidade relacionada a doenças do fígado. Esta pode ser caracterizada histologicamente por depósito de lipídios no interior dos hepatócitos, tornando-os disfuncionais (VERAS et al., 2018).

A DHGNA é o tipo mais comum de doença hepática no mundo que afeta adultos e crianças. Abrange um amplo espectro de doenças, desde esteatose simples em estágios iniciais até Esteato-Hepatite Não Alcoólica (EHNA) e fibrose, e eventualmente pode levar à cirrose e à carcinoma hepatocelular. Os fatores de risco mais frequentes para DHGNA são obesidade, diabetes mellitus tipo 2, dislipidemia, alguns medicamentos, glicocorticoides, esteroides anabolizantes, toxinas ambientais, síndrome da apneia do sono, hipotireoidismo, síndrome do ovário policístico e supercrescimento bacteriano no intestino delgado (SÁEZ-LARA et al., 2016; COTRIM et al., 2016).

A microbiota intestinal também pode desempenhar um papel fundamental no desenvolvimento e progressão de DHGNA. Bactérias patogênicas podem produzir endotoxinas em indivíduos com permeabilidade intestinal prejudicada. Estas podem atingir a circulação, levando a respostas inflamatórias extra e intra-hepáticas, que, por sua vez, induzem à progressão de DHGHA para EHNA. Estudos relatam que os pacientes afetados pela DHGNA possuem alta prevalência de supercrescimento bacteriano do intestino delgado e aumento da permeabilidade intestinal, expondo o fígado a metabólitos microbianos tóxicos (NOBILI et al., 2018).
Nos últimos anos, a literatura científica tem dedicado cada vez mais atenção ao eixo intestino-fígado, com estudos envolvendo a modulação da microbiota com o uso de probióticos e simbióticos; estes têm sido considerados promissores no tratamento da DHGNA por regularem a microbiota intestinal, modificarem a função da barreira intestinal e apresentarem efeitos imunomodulatórios, anti-inflamatórios e metabólicos, melhorando os parâmetros inflamatórios e reduzindo os marcadores de estresse oxidativo (FEROLLA, 2017).

Diante do exposto, o objetivo deste estudo foi realizar uma revisão sistemática acerca das evidências científicas sobre os efeitos da suplementação de probióticos e simbióticos em portadores da doença hepática gordurosa não alcoólica.

\section{MÉTODO}

Para alcançar o objetivo proposto foi realizada uma revisão sistemática, que é uma forma rigorosa de resumir as evidências científicas disponíveis que são derivadas de vários ensaios clínicos, estudos de diagnóstico e prognóstico, ou de um método em particular (ROEVER, 2017),

Para definição da questão norteadora, utilizou-se a estratégia Pico, segundo Santos, Pimenta e Nobre (2007), que representa $P=$ pacientes (portadores de doença hepática gordurosa não alcoólica), I = intervenção (suplementação de probióticos ou simbióticos), C =comparação (controle ou placebo), $\mathrm{O}=\mathrm{Ou}$ tcomes (desfechos). Elaborou-se a seguinte questão: "Quais os efeitos da suplementação de probióticos e simbióticos na doença hepática gordurosa não alcoólica?"

As fontes de dados foram as bases de dados MedLine via PubMed, Web Of Science e Scopus mediante os seguintes descritores: non-alcoholic fatty liver disease, probiotics, synbiotic, de acordo com os Descritores em Ciências da Saúde (DeCS) e do Medical Subject Headings (MeSH) como estratégia de busca. Os operadores booleanos (AND e/ou OR) foram utilizados nas bases de dados para garantir melhores resultados. A busca nas bases de dados foi feita por duas pesquisadoras de forma independente.

Como critérios de inclusão foram selecionados estudos primários publicados no período de 2016 a 2020 disponíveis na íntegra nas bases de dados selecionadas, e pesquisas que abordem sobre a suplementação de probióticos e simbióticos na doença hepática gordurosa não alcoólica no idioma inglês. Foram excluídos estudos não pertinentes ao tema e pesquisas de revisão bibliográfica, sistemática ou metanálise. 
Os dados coletados foram submetidos a uma leitura minuciosa para seleção. A qualidade dos estudos foi avaliada com base no delineamento e na coleta de dados descrita na metodologia, e as análises foram conduzidas e expostas em todos os vieses. As fontes potenciais foram avaliadas por meio do método de avaliação duplo cego, SJR e Qualis. Em seguida, os resultados foram apresentados em forma de tabe- las. O levantamento bibliográfico foi realizado em junho de 2020 nas bases de dados eletrônicas MedLine via PubMed, Web Of Science e Scopus.

Os descritores controlados foram selecionados após consulta aos termos Descritores em Ciência da Saúde (DeCs) e Medical Subject Headings (MeSH), conforme descrito e apresentado, juntamente com a estratégia de busca, no Quadro 1.

Quadro 1 - Descritores controlados utilizados para a construção da estratégia de busca nas bases MedLine via PubMed, Web Of Science e Scopus

\begin{tabular}{|l|l|}
\hline Base de Dados & Estratégia de Busca \\
\hline Web of Science & $\begin{array}{l}\text { TOPIC: (Non-alcoholic Fatty Liver Disease AND Probiotics OR Non-alcoholic Fatty Liver Disease AND } \\
\text { Synbiotic) } \\
\text { Refined by: DOCUMENT TYPES: (ARTICLE) AND PUBLICATION YEARS: (2020 OR } 2019 \text { OR } 2018 \text { OR } 2017 \text { OR } \\
\text { 2016) } \\
\text { Timespan: All years. Indexes: SCI-EXPANDED, SSCI, A\&HCI, CPCI-S, CPCI-SSH, ESCI. }\end{array}$ \\
\hline SCOPUS & $\begin{array}{l}\text { TITLE-ABS-KEY (non-alcoholic AND fatty AND liver AND disease AND probiotics OR synbiotic) AND (LIMIT- } \\
\text { TO (PUBYEAR, 2020) OR LIMIT-TO (PUBYEAR, 2019) OR LIMIT-TO (PUBYEAR, 2018) OR LIMIT-TO (PUBYEAR, } \\
\text { 2017) OR LIMIT-TO (PUBYEAR, 2016) AND (LIMIT-TO (DOCTYPE, “ar”)) AND (LIMIT-TO (EXACTKEYWORD, } \\
\text { “Human")) }\end{array}$ \\
\hline PUBMED & $\begin{array}{l}\text { Non-alcoholic Fatty Liver Disease AND Probiotics OR Synbiotic. Filters applied: Full text, Clinical Trial, } \\
\text { Randomized Controlled Trial, in the last 5 years, Humans, MEDLINE. }\end{array}$ \\
\hline
\end{tabular}

Fonte: As autoras, 2020.

A busca totalizou 2.231 produções, e, após a aplicação dos filtros (últimos 5 anos, 2016, 2017, $2018,2019,2020$, seres humanos, ensaio randomizado controlado, ensaio clínico, artigo, texto completo, Medline), 207 foram selecionadas, das quais foram excluídas pelo título e após leitura do resumo, 192 artigos. Resultaram 12 artigos que compuseram a amostra dos analisados. A Figura 1 (Prisma Flow) descreve o percurso realizado para a seleção dos estudos, segundo a base consultada.

Conforme os descritores utilizados na pesquisa, identificou-se no MedLine via PubMed 1.680 artigos, no Web Of Science 157 artigos e no Scopus 394. Na Figura 1 segue a estratégia de busca após a aplicação dos filtros Fluxograma (Prisma Flow).

A revisão cumpriu criteriosamente as seguintes etapas: formulação da questão norteadora; seleção de artigos tendo como base o ano de publicação e tí- tulo; seleção dos artigos por seus resumos e seleção pelo texto na íntegra e, logo após, extração dos dados dos estudos incluídos; avaliação e interpretação dos resultados; e, por fim, apresentação da revisão do conhecimento produzido.

\section{RESULTADOS}

A extração dos dados foi realizada com auxílio de instrumento próprio, contendo informações sobre autores, ano de publicação, tipo de estudo, país, periódico, banco de dados, qualis, SJR, população, intervenção e resultados mais relevantes. As produções selecionadas foram organizadas em tabelas de acordo com as variáveis identificadas.

Na Tabela 1 estão apresentados os resultados referentes à caracterização das produções incluídas na revisão quanto ao autor, ano de publicação, tipo de estudo, país, banco de dados, qualis e SJR. 
Efeitos da Suplementação de Probióticos e Simbióticos na Doença Hepática Gordurosa Não Alcoólica: Revisão Sistemática

Figura 1 - Fluxograma da seleção dos artigos

(Prisma Flow)
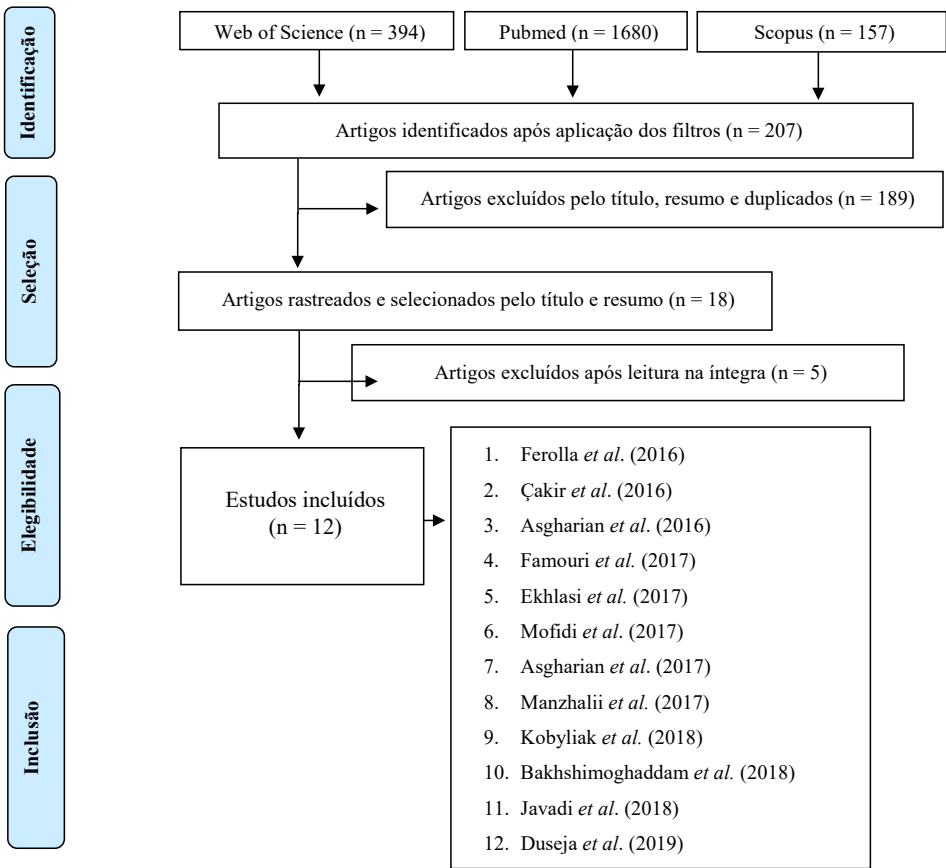

Fonte: As autoras, 2020. Adaptação de MOHER et al. (2011).

Tabela 1 - Distribuição dos resultados referentes aos estudos analisados sobre a suplementação de probióticos e simbióticos na doença hepática gordurosa não alcoólica, segundo autor/ano de publicação, tipo de estudo/país, periódico, banco de dados, Qualis e SJR

\begin{tabular}{|c|c|c|c|c|c|}
\hline $\begin{array}{l}\text { Autor/ ano de } \\
\text { publicação }\end{array}$ & Tipo de estudo/país & Periódico & Banco de dados & Qualis & SJR \\
\hline Ferolla et al. (2016) & Ensaio clínico controlado/Brasil & Nutrients & $\begin{array}{l}\text { Pubmed/Web } \\
\text { Science/Scopus }\end{array}$ & A1 & Q1:1.49 \\
\hline $\begin{array}{l}\text { Asgharian et al. } \\
(2016)\end{array}$ & $\begin{array}{l}\text { Ensaio clínico randomizado, duplo- } \\
\text { cego e controlado por placebo//rã }\end{array}$ & \begin{tabular}{|l|} 
International Journal of \\
Preventive Medicine
\end{tabular} & $\begin{array}{l}\text { Web of science/ } \\
\text { Pubmed }\end{array}$ & B1 & Q2:0.51 \\
\hline Famouri et al. (2017) & $\begin{array}{l}\text { Ensaio clínico randomizado, triplo- } \\
\text { cego, controlado por placebo/Irã }\end{array}$ & $\begin{array}{|lr|}\text { Journal of } & \text { Pediatric } \\
\text { Gastroenterology } & \text { and } \\
\text { Nutrition } & \\
\end{array}$ & $\begin{array}{l}\text { Pubmed/Web of } \\
\text { Science/Scopus }\end{array}$ & B1 & Q1:1.18 \\
\hline Çakir et al. (2017) & Ensaio clínico controlado/ Turquia & \begin{tabular}{|l|l|}
$\begin{array}{l}\text { Turkish Society of } \\
\text { Gastroenterology }\end{array}$ \\
\end{tabular} & \begin{tabular}{|l|}
$\begin{array}{l}\text { Pubmed/Web of } \\
\text { Science/Scopus }\end{array}$ \\
\end{tabular} & - & Q3:0.33 \\
\hline Ekhlasi et al. (2017) & $\begin{array}{l}\text { Ensaio clínico randomizado, } \\
\text { duplo-cego (Irã) }\end{array}$ & EXCLI Journal & $\begin{array}{l}\text { Web of Science/ } \\
\text { Pubmed/Scopus }\end{array}$ & B2 & Q2:0.59 \\
\hline Mofidi et al. (2017) & $\begin{array}{l}\text { Ensaio clínico randomizado, duplo- } \\
\text { cego, controlado por placebo/Irã }\end{array}$ & British Journal of Nutrition & $\begin{array}{l}\text { Pubmed/Web of } \\
\text { Science/Scopus }\end{array}$ & A1 & Q1:1.24 \\
\hline $\begin{array}{l}\text { Asgharian et al. } \\
(2017)\end{array}$ & $\begin{array}{l}\text { Ensaio clínico randomizado, duplo- } \\
\text { cego, controlado por placebo/Irã }\end{array}$ & $\begin{array}{l}\text { Iranian Red Crescent Medical } \\
\text { Journal }\end{array}$ & \begin{tabular}{|l|}
$\begin{array}{l}\text { Web of Science/ } \\
\text { Scopus }\end{array}$ \\
\end{tabular} & - & Q3:0.28 \\
\hline \begin{tabular}{lll|}
$\begin{array}{l}\text { Manzhalii et } \\
(2017)\end{array}$ & & \\
\end{tabular} & $\begin{array}{l}\text { Ensaio clínico, randomizado, } \\
\text { controlado e não cego/Ucrânia }\end{array}$ & Journal of Digestive Diseases & $\begin{array}{|ll|}\begin{array}{l}\text { Pubmed/Web of } \\
\text { Science/Scopus }\end{array} \\
\end{array}$ & - & Q2:0.66 \\
\hline Kobyliak et al. (2018) & $\begin{array}{l}\text { Ensaio clínico controlado por } \\
\text { placebo, duplo-cego/Ucrânia }\end{array}$ & \begin{tabular}{|l|} 
Journal of Gastrointestinal \\
and Liver Diseases
\end{tabular} & $\begin{array}{l}\text { Pubmed/Web } \\
\text { Science/Scopus }\end{array}$ & B2 & Q2:0.68 \\
\hline $\begin{array}{l}\text { Bakhshimoghaddam } \\
\text { et al. (2018) }\end{array}$ & $\begin{array}{l}\text { Ensaio clínico randomizado } \\
\text { controlado/Irã }\end{array}$ & The Journal of nutrition & Pubmed & A1 & Q1:1.8 \\
\hline Javadi et al. (2018) & $\begin{array}{l}\text { Ensaio clínico randomizado, duplo- } \\
\text { cego, controlado por placebo/Irã }\end{array}$ & $\begin{array}{l}\text { Asia Pacific Journal of Clinical } \\
\text { Nutrition }\end{array}$ & $\begin{array}{l}\text { Web of Science/ } \\
\text { Pubmed/Scopus }\end{array}$ & B2 & Q3:0.44 \\
\hline Duseja et al. (2019) & \begin{tabular}{|l|} 
Estudo clínico randomizado, \\
duplo-cego/Índia
\end{tabular} & BMJ Open Gastroenterology & Scopus/Pubmed & - & Q1:1.21 \\
\hline
\end{tabular}


De acordo com a Tabela 1, observa-se que $50 \%$ da produção científica sobre a temática estudada foi publicada em 2017, e o tipo de estudo mais utilizado foi o ensaio clínico randomizado (75\%). Em relação aos periódicos que publicaram artigos sobre a temática, observou-se uma dispersão, pois os estudos foram publicados em diferentes periódicos. O país que mais realizou pesquisas sobre os efeitos dos probióticos e simbióticos na DHGNA foi o Irã (58,3\%).
Levando em consideração o Qualis Capes (sistema brasileiro de avaliação de periódicos) e o SJR (indicador de prestígio dos periódicos científicos), observa-se que $25 \%$ foram classificadas como $A 1,41,7 \%$ como $B$, sendo $16,7 \%$ B1 e $25 \%$ B2, entretanto $33,3 \%$ dos periódicos não estavam classificados no sistema Qualis Capes. Em relação ao SJR, 41,7\% dos periódicos foram classificados como Q1, e os outros obtiveram classificação Q2 (33,3) e Q3 (25\%).

Tabela 2 - Distribuição dos resultados referentes aos estudos analisados sobre a suplementação de probióticos e simbióticos na doença hepática gordurosa não alcoólica, segundo a população do estudo, intervenção e principais resultados

\begin{tabular}{|c|c|c|c|}
\hline $\begin{array}{c}\text { Autor/ano de } \\
\text { publicação }\end{array}$ & População & Intervenção & Principais resultados \\
\hline Ferolla et al. (2016) & $\begin{array}{l}50 \text { pacientes com } \\
\text { EHNA divididos } \\
\text { aleatoriamente em } \\
\text { dois grupos: simbiótico } \\
\text { ( } \mathrm{n}=27 \text { ) e } 23 \text { placebo } \\
\text { ( } \mathrm{n}=23 \text { ) } \\
\text { Faixa etária: } 25 \text { a } 74 \\
\text { anos } \\
\text { (Idade média: } 57,3 \\
\text { anos). }\end{array}$ & $\begin{array}{l}\text { O grupo de estudo recebeu } 5 \text { g do simbiótico que } \\
\text { consistia em } 4 \text { g de fibra dietética (goma guar } \\
\text { parcialmente hidrolisada e inulina) e } 1 \times 10^{8} \text { UFC de } L \text {. } \\
\text { reuteri, duas vezes ao dia durante três meses. Todos os } \\
\text { participantes foram orientados e tiveram intervenção } \\
\text { dietética, receberam um plano alimentar (1500 kcal } \\
\text { para mulheres e } 1800 \text { kcal para homens) e instruções } \\
\text { nutricionais gerais sobre alimentação saudável } \\
\text { e equilibrada, com base no Guia Alimentar para } \\
\text { População Brasileira, e foram incentivados a manter } \\
\text { suas atividades físicas habituais durante o estudo. }\end{array}$ & $\begin{array}{l}\text { O grupo simbiótico apresentou } \\
\text { redução da esteatose hepática } \\
(p=0,027) \text {, peso }(p=0,006) \text {, IMC } \\
(p=0,005), C C(p=0,001) \text {, ácido } \\
\text { úrico }(p=0,006) \text {. }\end{array}$ \\
\hline $\begin{array}{l}\text { Asgharian et al. } \\
(2016)\end{array}$ & $\begin{array}{l}60 \text { adultos com } \\
\text { DGHNA divididos em } \\
\text { dois grupos: simbiótico } \\
(n=38) \text { e placebo } \\
(n=36) \text {. } \\
\text { Faixa etária: } 18 \text { a } 60 \\
\text { anos } \\
\text { (Média: } 46,57 \pm 1,7) \text {. }\end{array}$ & $\begin{array}{l}\text { O grupo simbiótico recebeu uma cápsula de } 500 \\
\text { mg/dia contendo sete espécies de probióticos } \\
\text { (Lactobacillus casei, Lactobacillus acidophilus, } \\
\text { Lactobacillus rhamnosus, Lactobacillus bulgaricus, } \\
\text { Bifidobacterium breve, Bifidobacterium longum, } \\
\text { Streptococcus thermophilus) e frutooligossacarídeos } \\
\text { durante } 8 \text { semanas. }\end{array}$ & $\begin{array}{l}\text { Após a intervenção os pacientes } \\
\text { com DHGNA não apresentaram } \\
\text { alterações significativas nos } \\
\text { níveis de PCR, ALT e AST em } \\
\text { comparação com dois grupos. } \\
\text { O grupo simbiótico, entretanto, } \\
\text { apresentou diminuição no grau da } \\
\text { DHGNA, 50\% e 25\% dos pacientes } \\
\text { com DGHNA leve (grau 1) e } \\
\text { moderado (grau 2) diminuíram } \\
\text { para grau zero, respectivamente. } \\
\text { Em } 43,8 \% \text { dos pacientes com } \\
\text { DGHNA moderada, a gravidade da } \\
\text { esteatose hepática reduziu para } \\
\text { o grau } 1 .\end{array}$ \\
\hline Famouri et al. (2017) & $\begin{array}{l}64 \text { crianças e } \\
\text { adolescentes obesas } \\
\text { com DHGNA divididos } \\
\text { em dois grupos: } \\
\text { probiótico }(\mathrm{n}=32) \text { e } \\
\text { placebo ( } \mathrm{n}=36) \\
\text { Faixa etária: } 10 \text { a } 18 \\
\text { anos } \\
\text { (Idade média: } \\
12,7 \pm 2,2)\end{array}$ & $\begin{array}{l}\text { O grupo probiótico recebeu uma cápsula/dia de } \\
\text { probióticos contendo: Lactobacillus acidophilus } \\
3 \times 10^{9} \text { UFC, Bifidobacterium lactis } 6 \times 10^{9} \text { UFC, } \\
\text { Bifidobacterium bifidum, } 2 \times 10^{9} \text { UFC, Lactobacillus } \\
\text { rhamnosus, } 2 \times 10^{9} \text { UFC durante } 12 \text { semanas. Hábitos } \\
\text { saudáveis de estilo de vida foram recomendados } \\
\text { para os participantes dos dois grupos. Estes foram } \\
\text { orientados a aumentar a ingestão de frutas e vegetais } \\
\text { e diminuir o consumo de fast-food e refeições com } \\
\text { alto teor de gordura e açúcar, e, além disso, foram } \\
\text { instruídos a aumentar suas atividades físicas diárias e } \\
\text { diminuir o uso de aparelhos eletrônicos. }\end{array}$ & $\begin{array}{l}\text { Após a intervenção os pacientes } \\
\text { com DHGNA apresentaram } \\
\text { diminuição da CC }(p=0,001), A L T \\
(p=0,002), C T(p<0,001) \text { e grau de } \\
\text { esteatose }(p<0,001) .\end{array}$ \\
\hline
\end{tabular}




\begin{tabular}{|c|c|c|c|}
\hline Çakir et al. (2017) & $\begin{array}{l}28 \text { crianças e } \\
\text { adolescentes com } \\
\text { DHGNA (grupo } \\
\text { simbiótico) e } 30 \\
\text { crianças e adolescente } \\
\text { saudáveis (grupo } \\
\text { controle). } \\
\text { Faixa etária: } \leq 18 \text { anos } \\
\text { (Idade média: } 12 \pm 2,2 \\
\text { anos) }\end{array}$ & $\begin{array}{l}\text { As crianças e adolescentes com DHGNA receberam } 1 \\
\text { cápsula/dia de simbiótico: } 7 \times 10^{9} \text { UFC de probióticos } \\
\text { ativos (Bifidobacterium lactis, Lactobacillus acidophilus } \\
\text { e Lactobacillus casei) e } 100 \text { mg de inulina de chicória, } \\
\text { durante } 4 \text { meses. Além da suplementação, foi prescrito } \\
\text { aos participantes uma dieta hipocalórica (com redução } \\
10 \%-20 \% \text { calorias) de acordo com a idade, } 50 \%-60 \% \\
\text { de carboidratos, } 20 \%-30 \% \text { de lipídios (dois terços } \\
\text { saturados e um terço insaturados) e } 10 \% \text { a } 20 \% \text { de } \\
\text { proteína, e um programa de exercício moderado } \\
\text { (exercício aeróbico de } 30-45 \text { min pelo menos } 3 \text { vezes } \\
\text { por semana). }\end{array}$ & $\begin{array}{l}\text { Após a intervenção os } \\
\text { participantes com DGHNA } \\
\text { apresentaram diminuição do } \\
\text { IMC }(p=0,001) \text {, percentual de } \\
\text { gordura }(p=0,001) \text {, escore-z do } \\
\text { IMC }(p=0,001), C T(p=0,005), \text { LDL } \\
(p=0,005), \text { TNF- } \alpha(p=0,01), \text { PCR } \\
\text { ( } p=0,003) \text {, etanol ( } p=0,001) \text { e } \\
\text { estado antioxidante total }(p=0,02) \text {. }\end{array}$ \\
\hline Ekhlasi et al. (2017) & $\begin{array}{l}60 \text { adultos com } \\
\text { DGHNA e IMC } 25 \\
\mathrm{~kg} / \mathrm{m}^{2} \text { a } 29,9 \mathrm{~kg} / \mathrm{m}^{2} \\
\text { divididos em quatro } \\
\text { grupos: simbiótico } \\
\text { ( } \mathrm{n}=15) \text {, simbiótico + } \\
\text { alfa-tocoferol }(\mathrm{n}=15) \text {, } \\
\text { alfa-tocoferol }(\mathrm{n}=15) \text { e } \\
\text { placebo ( } \mathrm{n}=15) . \\
\text { Faixa etária: } 25 \text { a } 64 \\
\text { anos } \\
\text { (Idade média: } 44 \pm 20 \\
\text { anos). }\end{array}$ & $\begin{array}{l}\text { O grupo simbiótico recebeu } 1 \text { cápsula simbiótica } \\
\text { contendo: Lactobacillus casei, Lactobacillus rhamnosus, } \\
\text { Stretococcus thermophilus, Bifidobacterium breve, } \\
\text { Lactobacillus acidophilus, Bifidobacterium longum, } \\
\text { Lactobacillus bulgaricus. A concentração de cada cepa } \\
\text { probiótica foi de } 2 \times 10^{8} \text { UFC e frutooligossacarídeos } \\
\text { (prebiótico). O grupo simbiótico + alfa-tocoferol } \\
\text { recebeu simbiótico + uma dose diária de } 400 \text { UI de } \\
\text { alfa-tocoferol. O grupo alfa-tocoferol recebeu } 400 \\
\text { UI de alfa-tocoferol. Todos os grupos receberam } \\
\text { suplementação por } 8 \text { semanas. }\end{array}$ & $\begin{array}{l}\text { Todos os grupos que tiveram } \\
\text { intervenção apresentaram } \\
\text { diminuição do TNF } \alpha \text {. A redução } \\
\text { nos níveis de TNF } \alpha \text { entre os } \\
\text { indivíduos que receberam } \\
\text { simbiótico e alfa-tocoferol foi } \\
\text { maior em comparação com os } \\
\text { outros grupos ( } P=0,001 \text { ). Foi } \\
\text { o único grupo que apresentou } \\
\text { redução do MDA ( } p<0.001 \text { ). } \\
\text { O grupo simbiótico e grupo } \\
\text { simbiótico + alfa temporal } \\
\text { apresentaram redução nos níveis } \\
\text { de ALT ( } p=0,005 ; p<0,001 \text { ), ALP } \\
\text { ( } p<0,001 ; p<0,001), A S T \text { ( } p<0,001 ; \\
p<0,001) .0 \text { grupo alfa-tocoferol } \\
\text { diminuiu AST ( } p=0,004 \text { ). }\end{array}$ \\
\hline Mofidi et al. (2017) & $\begin{array}{l}42 \text { adultos com } \\
\text { DGHNA com IMC: }> \\
25 \mathrm{~kg} / \mathrm{m}^{2} \text { divididos em } \\
\text { dois grupos: simbiótico } \\
(\mathrm{n}=38) \text { e placebo } \\
(\mathrm{n}=36) \text {. } \\
\text { Faixa etária: } \geq 18 \text { anos } \\
\text { (Média: } 44,45 \pm 10,69)\end{array}$ & $\begin{array}{l}\text { O grupo simbiótico recebeu } 2 \text { cápsulas/dia contendo: } \\
200 \text { milhões de probióticos de sete linhagens } \\
\text { (Lactobacillus casei, Lactobacillus rhamnosus, } \\
\text { Streptococcus thermophilus, Bifidobacterium breve, } \\
\text { Lactobacillus acidophilus, Lactobacillus acidophilus, } \\
\text { Bifidobacterium longum e Lactobacillus bulgaricus), } \\
125 \text { mg de frutooligossacarídeo (prebiótico) e estearato } \\
\text { de magnésio em cápsula vegetal } \\
\text { (hidroxipropilmetil celulose), durante } 28 \text { semanas. }\end{array}$ & $\begin{array}{l}\text { Após a intervenção, o grupo } \\
\text { simbiótico apresentou redução } \\
\text { da esteatose hepática placebo } \\
(p<0,001) \text {, fibrose hepática } \\
(p<0,001), \text { AST }(p=0,005) \text {, glicemia } \\
\text { em jejum }(p=0,05), \text { TG }(p<0,001) \text {, } \\
\text { PCR }(p=0,05), \text { NF-kB p65 ( } p \\
=0,05) .\end{array}$ \\
\hline $\begin{array}{l}\text { Asgharian et al. } \\
(2017)\end{array}$ & $\begin{array}{l}60 \text { adultos com } \\
\text { DGHNA divididos em } \\
\text { dois grupos: simbiótico } \\
(n=38) \text { e placebo } \\
(n=36) \text {. } \\
\text { Faixa etária: } 18 \text { a } 60 \\
\text { anos } \\
\text { (Média: } 46,6 \pm 1,7) \text {. } \\
\end{array}$ & $\begin{array}{l}\text { O grupo simbiótico recebeu uma cápsula de } 500 \\
\text { mg/dia contendo sete espécies de probióticos } \\
\text { (Lactobacillus casei, Lactobacillus acidophilus, } \\
\text { Lactobacillus rhamnosus, Lactobacillus bulgaricus, } \\
\text { Bifidobacterium breve, Bifidobacterium longum, } \\
\text { Streptococcus thermophilus) e frutooligossacarídeos } \\
\text { durante } 8 \text { semanas. }\end{array}$ & $\begin{array}{l}\text { Após a intervenção os pacientes } \\
\text { com DHGNA apresentaram } \\
\text { diminuição no peso }(p=0,001) \text {, } \\
\text { gordura corporal }(p=0,02), C T \text { ( } p \\
=0,04) \text {. }\end{array}$ \\
\hline $\begin{array}{l}\text { Manzhalii et al. } \\
(2017)\end{array}$ & $\begin{array}{l}75 \text { adultos com } \\
\text { EHNA divididos } \\
\text { aleatoriamente em } \\
\text { dois grupos: simbiótico } \\
\text { ( } n=38 \text { ) e controle } \\
\text { ( } n=37) \text {. } \\
\text { Faixa etária: } 30 \text { a } 60 \\
\text { anos } \\
\text { (Idade média: } \\
44,3 \pm 1,5) .\end{array}$ & $\begin{array}{l}\text { O grupo experimental (simbiótico) recebeu cápsulas } \\
\text { com Lactobacillus casei, Lactobacillus rhamnosus, } \\
\text { Lactobacillus bulgaricus, Bifidobacterium longum, } \\
\text { Streptococcus Thermophilus ( } 10^{8} \text { bactérias/cápsula } \\
\text { no total) e frutooligossacarídeos, uma vez ao dia } \\
\text { por } 12 \text { semanas. Ambos os grupos de pacientes } \\
\text { com NASH (grupo controle e o grupo experimental) } \\
\text { foram instruídos a manter uma dieta hipocalórica e } \\
\text { hipolipídica ( } 30 \text { a } 90 \text { g de gordura/dia e } 1800 \mathrm{kcal} / \mathrm{dia}) \text {. }\end{array}$ & $\begin{array}{l}\text { Os participantes do grupo } \\
\text { simbiótico após a intervenção } \\
\text { apresentaram diminuição da ALT } \\
(p<0,05), A S T(p<0,05) \text {, fibrose } \\
\text { hepática }(p<0,05), C T(p<0,05) \text { e } \\
\text { IMC }(p<0,05) \text { em comparação com } \\
\text { o grupo controle. }\end{array}$ \\
\hline
\end{tabular}




\begin{tabular}{|c|c|c|c|}
\hline Kobyliak et al. (2018) & $\begin{array}{l}58 \text { pacientes com } \\
\text { DHGNA, diabetes } \\
\text { mellitus tipo } 2 \mathrm{e} \\
\mathrm{IMC} \geq 25 \mathrm{~kg} / \mathrm{m}^{2} \\
\text { foram divididos } \\
\text { aleatoriamente em } \\
\text { dois grupos: probiótico } \\
\text { ( } \mathrm{n}=30) \text { ou placebo } \\
(\mathrm{n}=28) . \\
\text { Faixa etária: } 18 \text { a } 65 \\
\text { anos } \\
\text { (Idade média: } \\
53,4 \pm 9,55 \text { anos). } \\
\end{array}$ & $\begin{array}{l}\text { O grupo probiótico recebeu um sachê de } 10 \mathrm{~g} \\
\text { ao dia composto de } 14 \text { cepas probióticas vivas } \\
\text { de Lactobacillus }+ \text { Lactococos }\left(6 \times 10^{10} \mathrm{UFC} / \mathrm{g}\right) \text {, } \\
\text { Bifidobacterium }\left(1 \times 10^{10} / \mathrm{g}\right) \text {, Propionibacterium (3 } \\
\times 10^{10} / \mathrm{g} \text { ) e gênero Acetobacter }\left(1 \times 10^{6} / \mathrm{g}\right) \text { durante } 8 \\
\text { semanas. Duas semanas antes do início do estudo, os } \\
\text { pacientes foram instruídos a sessões individuais com } \\
\text { um nutricionista para seguir um tratamento dieta } \\
\text { de mudança de estilo de vida, conforme o National } \\
\text { Colesterol Education Program (NCEP). Além disso, } \\
\text { os participantes foram instruídos a continuar com } \\
\text { um tratamento anti-hiperglicêmico e receberam } \\
\text { treinamento físico leve padronizado } 1 \text { hora por dia. }\end{array}$ & $\begin{array}{l}\text { No grupo probiótico após a } \\
\text { intervenção houve diminuição } \\
\text { do índice hepático de gordura } \\
(p<0,001), \text { AST }(p<0,001), \text { GGT } \\
(p=0,001), \text { TG }(p=0,001), \text { CT } \\
(p=0,001) \text {, LDL }(p=0,004), \text { VLDL ( } p \\
=0,033) \text {, TNF- } \alpha(p<0,001) \text { e IL-6 } \\
(p=0,003)\end{array}$ \\
\hline $\begin{array}{l}\text { Bakhshimoghaddam } \\
\text { et al. (2018) }\end{array}$ & $\begin{array}{l}102 \text { participantes } \\
\text { DHGNA divididos em } \\
\text { três grupos: iogurte } \\
\text { simbiótico }(n=34), \\
\text { grupo de iogurte } \\
\text { convencional }(n=34) \\
\text { e grupo de controle } \\
\text { ( } n=34) \\
\text { Faixa etária: } \geq 18 \text { anos } \\
\text { (Média: } 38,8 \pm 9,0) .\end{array}$ & $\begin{array}{l}\text { Os grupos de intervenção consumiram } 300 \text { g de iogurte } \\
\text { simbiótico ou iogurte convencional diariamente por } 24 \\
\text { semanas e receberam orientação para seguirem um } \\
\text { estilo de vida saudável, de acordo com as diretrizes } \\
\text { clínicas para sobrepeso e obesidade em adultos do } \\
\text { National Institutes of Health (NIH) e da Associação } \\
\text { Norte-Americana para o Estudo de obesidade. O } \\
\text { grupo controle foi aconselhado a seguir um estilo } \\
\text { de vida saudável por } 24 \text { semanas. Toda semana os } \\
\text { participantes recebiam iogurtes convencionais ou } \\
\text { simbióticos com 1,4\% de gordura. Em ambos tipos de } \\
\text { iogurte, culturas iniciais de Streptococcus thermophilus } \\
\text { e Lactobacillus delbrueckii subsp. Bulgaricus foram } \\
\text { utilizadas. O iogurte simbiótico também continha } 10^{8} \\
\text { UFC/mL Bifidobacterium animalis subsp. Lactis como } \\
\text { probiótico e 1,5 g de inulina como prebiótico. }\end{array}$ & $\begin{array}{l}\text { O grupo iogurte simbiótico, } \\
\text { após a intervenção, apresentou } \\
\text { diminuição no grau da DHGNA } \\
\text { ( } p<0,001) \text {, em comparação com } \\
\text { os outros grupos. As alterações } \\
\text { nas concentrações séricas de ALT } \\
\text { ( } p=0,008), \text { AST ( } p<0,001), \text { ALP } \\
\text { ( } p=0,024) \text { e GGT }(P<0,001) \text { foram } \\
\text { significativamente diferentes } \\
\text { entre os três grupos. O grupo } \\
\text { simbiótico mostrou uma redução } \\
\text { maior nas concentrações séricas } \\
\text { dessas enzimas do que os grupos } \\
\text { convencional e controle. O CT, } \\
\text { TG, estado de oxidante total } \\
\text { diminuíram significativamente } \\
\text { e CTRP-5, GLP-2, capacidade } \\
\text { antioxidante total e QUICKI } \\
\text { aumentaram significativamente } \\
\text { no grupo simbiótico comparado } \\
\text { com o convencional. } \\
\end{array}$ \\
\hline Javadi et al. (2018) & $\begin{array}{l}75 \text { adultos com } \\
\text { DGHNA divididos em } \\
4 \text { grupos: probiótico } \\
\text { ( } n=20), \text { prebiótico } \\
\text { ( } n=19), \text { probiótico + } \\
\text { prebiótico }(n=17) \text { e } \\
\text { placebo }(n=19) . \\
\text { Faixa etária: } 20 \text { a } 60 \\
\text { anos } \\
\text { (Idade média: } \\
42,00 \pm 8,95) . \\
\end{array}$ & $\begin{array}{l}\text { O grupo probiótico recebeu } 1 \text { cápsula/dia de probiótico } \\
\text { contendo: Bifidobacterium longum, Lactobacillus } \\
\text { acidophilus } 2 \times 10^{7} \text { UFC/dia. O grupo prebiótico } \\
\text { recebeu um sachê } 10 \text { g de inulina. Grupo probiótico e } \\
\text { prebiótico recebeu } 1 \text { sachê contendo: Bifidobacterium } \\
\text { longum, Lactobacillus acidophilus } 2 \times 10^{7} \text { UFC/dia e } 10 \\
\text { g de inulina. Todos os tratamentos foram ministrados } \\
\text { por } 3 \text { meses. }\end{array}$ & $\begin{array}{l}\text { O grupo probiótico + prebiótico } \\
\text { após a suplementação de três } \\
\text { meses apresentou redução } \\
\text { significativa na PCR }(p=0,01), \text { MDA } \\
(p=0,01) \text {, capacidade antioxidante } \\
\text { total }(p=0,01) \text {. O grupo } \\
\text { prebióticco apresentou apenas } \\
\text { diminuição do MDA ( } p=0,01) . \\
\text { O grupo probiótico diminuiu } \\
\text { MDA ( } p=0,01) \text { e a capacidade } \\
\text { antioxidante total }(p=0,01) . \\
\end{array}$ \\
\hline Duseja et al. (2019) & $\begin{array}{l}30 \text { pacientes com } \\
\text { DGHNA foram } \\
\text { randomizados para } \\
\text { receber probiótico } \\
(\mathrm{n}=17) \text { ou placebo } \\
(\mathrm{n}=13) \text {. } \\
\text { Faixa etária: } \geq 18 \text { anos } \\
\text { (Idade média: } 38 \pm 10 \\
\text { anos). }\end{array}$ & $\begin{array}{l}\text { O grupo probiótico recebeu } 2 \text { cápsulas ao dia (cada } \\
\text { cápsula continha 112,5 bilhões de bactérias vivas, } \\
\text { liofilizadas, lácticas e bifidobactérias, lactobacillus } \\
\text { paracasei, lactobacillus plantarum, lactobacillus } \\
\text { acidophiluse lactobacillus delbrueckii subsp. Bulgaricus, } \\
\text { bifidobacterium longum, bifidobacterium infantis, } \\
\text { bifidobacterium breve e streptococcus thermophilus) } \\
\text { durante } 12 \text { meses. Todos os pacientes foram } \\
\text { orientados a realizar modificações no estilo de vida } \\
\text { e aconselhados a fazer exercícios físicos regulares. } \\
\text { Pacientes com sobrepeso ou obesidade foram } \\
\text { orientados a reduzir } 5 \% \text { a } 10 \% \text { do peso ( } \leq 1,6 \mathrm{~kg} / \\
\text { semana) com a ajuda de uma dieta hipocalórica com } \\
\text { redução de } 30 \% \text { na ingestão de calorias, diminuindo a } \\
\text { ingestão de carboidratos e gorduras. }\end{array}$ & $\begin{array}{l}\text { O grupo probiótico, após a } \\
\text { suplementação, diminuiu escore } \\
\text { de atividade da DHGNA ( } p=0,004) \text {, } \\
\text { balonamento hepatocelular } \\
\text { ( } p=0,05) \text {, fibrose hepática } \\
(p=0,018) \text { e ALT }(p=0,046) \text {, leptina } \\
(p=0,006) \text {, TNF- } \alpha(p=0,016) \text { e } \\
\text { endotoxinas ( } p=0,017) \text { e melhora } \\
\text { na classificação da esteatose } \\
\text { hepática }(p=0,026) \text {. }\end{array}$ \\
\hline
\end{tabular}

Legenda: ALT: alanina aminotransferase; AST: aspartato aminotransferase; CC: circunferência da cintura; CT: colesterol total; CTRP-5: proteína 5 relacionada ao TNF do complemento-c1q; DHGNA: doença hepática gordurosa não alcoólica; EHNA: esteato-hepatite não alcoólica; GGT: gamaglutamiltransferase; GLP-2: peptídeo semelhante ao glucagon-2; IL-6: interleucina 6; IMC: Índice de massa corporal; LDL: lipoproteínas de baixa densidade; MDA: malondialdeído; NF-kb: factor nuclear kappa beta - proteína 65; PCR: proteína C reativa; QUICKI: sensibilidade quantitativa à insulina; TG: triglicerídeos; TNF- $\alpha$ : fator de necrose tumoral; UFC: unidade formadora de colônia; VLDL: lipoproteína de muito baixa densidade. 
A Tabela 2 expõe a distribuição dos estudos selecionados, apresentando as produções científicas acerca da eficácia da suplementação de probióticos e simbióticos em pacientes com doença hepática gordurosa não alcoólica. A população nos estudos variou de 30 a 138 participantes, com tempo de intervenção de 8 semanas a 12 meses. Os parâmetros mais avaliados foram as enzimas hepáticas (alanina aminotransferase, aspartato aminotransferase, gamaglutamiltransferase), perfil lipídico (colesterol total, lipoproteínas de baixa densidade, triglicerídeos), fator de necrose tumoral, grau de esteatose, fibrose hepática e índice de gordura corporal, entretanto nem todos os estudos avaliaram esses parâmetros de maneira primária.

Os outros parâmetros avaliados com menor frequência foram: peso, percentual de gordura, lipoproteína de muito baixa densidade, endotoxinas, interleucina 6 , leptina, proteína $C$ reativa, ácido úrico, capacidade/estado antioxidante total, malondialdeído, factor nuclear kappa beta - proteína 65 , glicemia em jejum, sensibilidade quantitativa à insulina e proteína 5 relacionada ao TNF do complemento-c1q.

As cepas probióticas do gênero Lactobacillus e gênero Bifidobacterium foram usadas em todos os estudos, seguidas do Streptococcus thermophilus em sete estudos. Utilizaram também os gêneros Lactococcus e Acetobacter, com apenas um estudo cada. A dose variou de $10^{7}$ a $10^{10}$ unidades formadoras de colônia (UFC). Os prebióticos mais empregados na composição dos simbióticos foram frutooligossacarídeos em cinco estudos, inulina em três pesquisas e apenas um estudo utilizou goma guar.

\section{DISCUSSÃO}

A DHGNA é considerada a manifestação hepática da síndrome metabólica, afetando, atualmente, aproximadamente $25 \%$ dos adultos da população mundial e quase $70 \%$ dos pacientes com diabetes mellitus tipo 2. A DHGNA ocorre com mais frequência em homens do que em mulheres, e geralmente afeta adultos de meia-idade e idosos em razão de os principais fatores de risco (sobrepeso, obesidade, diabetes tipo 2, dislipidemia e sedentarismo), associados ao seu desenvolvimento e progressão, aumentarem a prevalência com o avanço da idade (MANTOVANI; TARGHER; ZOPPINI, 2020).

Em crianças e adolescentes a etiologia mais comum da DHGNA é a obesidade, aproximando-se de $10 \%$ da população pediátrica geral e $34 \%$ na população pediátrica obesa, principalmente nos países industrializados. Além da obesidade, a suscetibilida- de genética e fatores ambientais são sugeridos como causas precoces da DHGNA. Em razão da lesão no tecido hepático em decorrência da doença, as crianças e adolescentes com DGHNA afetados podem apresentar a doença hepática avançada mais cedo na idade adulta e comorbidades, como a síndrome metabólica e doença cardiovascular ao longo da vida (GOLDNER; LAVINE, 2020).

Já Tagliari et al. (2017) afirmam que o desenvolvimento da DHGNA envolve duas fases: na primeira fase ocorre o armazenamento inadequado de lipídios ou acúmulo ectópico no fígado; na segunda fase o estresse oxidativo provoca lesão dos hepatócitos e leva ao desenvolvimento da inflamação. Em decorrência da alteração fisiológica do fígado, células inflamatórias são ativadas, ocorrendo alterações dos mecanismos de controle e da ativação dos fatores derivados do intestino, ocasionando o desequilíbrio na microbiota intestinal.

De acordo com Ferolla et al. (2016), a microbiota intestinal pode desempenhar um papel na patogênese da esteatose hepática em decorrência da liberação de lipopolissacarídeo (LPS), uma endotoxina que aumenta a produção de etanol e que ativa citocinas inflamatórias nas células epiteliais luminais e macrófagos hepáticos. Segundo Kobyliak et al. (2018), o LPS, ao ativar as citocinas inflamatórias, resulta na produção de espécies de radicais livres no fígado que contribuem para o desenvolvimento e progressão da DHGNA e EHNA. Em virtude de as endotoxinas ativarem as células de Kupffer presentes no fígado, como consequência ocorre o aumento da produção de TNF- $\alpha$ e IL-6, que contribuem para o início da fibrose hepática.

A realização da modulação da microbiota intestinal com prebióticos (carboidratos não digeríveis, com efeitos benéficos, pois estimulam seletivamente o crescimento e/ou a atividade das bactérias gram-positivas e suprimem as bactérias gram-negativas residentes no cólon), probióticos (microrganismos vivos que, quando consumidos em quantidades adequadas, conferem benefício à saúde do hospedeiro) e simbióticos (combinação de probióticos e prebióticos) estimula seletivamente o crescimento, ativando o metabolismo de bactérias promotoras de saúde e exercendo efeitos benéficos na progressão da DHGNA (MA; ZHOU; LI, 2017; FEROLLA, 2017).

Nesse sentido, Famouri et al. (2017) avaliaram a suplementação com probióticos e mudança no estilo de vida em crianças e adolescentes obesos com DHGNA. Após a intervenção houve redução significativa do grau de esteatose no grupo intervenção (53\%) em relação ao grupo placebo $(16,5 \%)$, conforme a ultras- 
sonografia. O probiótico utilizado no presente estudo reduziu as concentrações de AST e ALT, e os níveis de ambos os marcadores foram significativamente mais baixos no grupo probiótico do que no grupo placebo. O composto probiótico utilizado no presente estudo teve efeito significativo na $\mathrm{CC}$, mas não houve alterações significativas no peso e no IMC. Segundo os autores, na literatura os resultados são controversos em relação ao impacto dos probióticos no IMC e na CC. Alguns estudos indicaram efeito benéfico, enquanto outros não confirmaram a melhora do perfil antropométrico.

Resultados semelhantes observados por Kobyliak et al. (2018) ao avaliarem os benefícios da suplementação de probióticos em adultos com DHGNA e IMC $\geq 25 \mathrm{~kg} / \mathrm{m}^{2}$, identificaram, após a intervenção, que ocorreu redução significativa do índice hepático de gordura em comparação com o grupo placebo nos desfechos secundários. Os probióticos reduziram significativamente a atividade de AST e GGT, mas não houve alteração de ALT em relação aos marcadores de inflamação sistêmica crônica. Apenas os níveis de TNF- $\alpha$ e IL- 6 mudaram significativamente entre grupos. Os participantes, entretanto, eram portadores de diabetes mellitus tipo 2 além DHGNA, diferentemente dos outros estudos avaliados.

De acordo com Sweet, Khoo e Nguyen (2017), o diagnóstico de DHGNA requer confirmação da esteatose mediante ultrassonografia ou biópsia na ausência de uso significativo de álcool e exclusão de outras doenças, como hepatite viral, hemocromatose, doença de Wilson, autoimunes e lesão hepática iatrogênica. O diagnóstico é geralmente sugerido em pacientes assintomáticos, com base nos níveis séricos elevados das enzimas hepáticas na triagem de exames laboratoriais, sendo solicitadas imagens adicionais para avaliar a presença de esteatose ou fibrose. Em estágios mais avançados, a biópsia é necessária para determinar a gravidade do dano hepático com base no grau ou estágio patológico.

Nesse aspecto, a pesquisa de Duseja et al. (2019), após o término da suplementação de probióticos, avaliou, por meio de biópsia, a eficácia dos probióticos na melhoria da histologia hepática dos adultos com DHGNA. O grupo probiótico diminuiu significativamente o escore de atividade da DHGNA, balonamento hepatocelular e fibrose hepática. Além das melhorias na histologia hepática, os pacientes do grupo probiótico apresentaram maior redução na ALT sérica e melhora no perfil de citocinas (TNF- $\alpha$ e endotoxinas) em comparação aos pacientes que receberam placebo. Não houve, entretanto, alteração significati- va no peso corporal ou nos componentes da síndrome metabólica (perfil lipídico, hipertensão e glicemia em jejum).

O estudo de Çakir et al. (2017) comparou crianças saudáveis com crianças com DHGNA. Ao avaliar os parâmetros antropométricos, enzimas hepáticas e perfil lipídico, pacientes com DHGNA tinham elevados níveis de etanol, inflamação sistêmica e permeabilidade intestinal quando comparados a crianças saudáveis. Após a suplementação com simbióticos e mudanças de estilo de vida, as crianças com DHGNA apresentaram diminuição significativa do IMC, percentual de gordura, escore-z do IMC, colesterol total, $L D L, T N F-\alpha, P C R$, etanol e estado antioxidante total. Ao avaliar as enzimas hepáticas ALT e AST, no entanto, a diminuição, após 4 meses de intervenção, não foi significativa. Corroborando a pesquisa de Asgharian et al. (2016) com adultos com DHGNA, após 8 semanas de suplementação de probióticos não houve alterações significativas nos níveis de ALT, AST e PCR, no entanto verificou-se melhora do grau de esteatose. $O$ grupo simbiótico apresentou diminuição no grau da DHGNA, e 50\% dos indivíduos com grau 1 diminuíram para grau zero após o uso de simbióticos.

Conforme Scheid et al. (2018), a elevação das aminotransferases na DHGNA ocorre em decorrência da necessidade de reações aumentadas de transaminação para lidar com o distúrbio metabólico hepático (o acúmulo anormal de triglicerídeos), também associado ao aumento da gliconeogênese e resistência à insulina. O fígado, para manter a homeostase, aumenta positivamente ASL e ALT, levando a mudanças nas quantidades de aminoácidos liberados na circulação.

Sato et al. (2015) citam que o estresse oxidativo é considerado um dos fatores principais no aparecimento e desenvolvimento da DHGNA para EHNA, e a terapia antioxidante é um tratamento promissor, principalmente com a suplementação de vitamina E. Acredita-se que esta atue como um agente antioxidante e possibilite efeitos benéficos. Ekhlasi et al. (2017), avaliaram a suplementação isolada de simbióticos, alfa-tocoferol e associação destes por 8 semanas em pacientes com DHGNA. Após a intervenção, os dois grupos (grupo simbiótico e grupo simbiótico+alfa tocoferol) que receberam simbiótico apresentaram redução significativa nas enzimas hepáticas (ALT, ALP, AST), TNF $\alpha$ e pressão arterial sistólica em comparação com o grupo controle. Nos níveis de óxido nítrico e pressão sanguínea diastólica não apresentaram alterações significativas. 
Dois estudos presentes na Tabela 2 avaliaram a suplementação de simbiótico na EHNA um estágio mais avançado da DHGNA. Na pesquisa de Ferolla et al. (2016), adultos com EHNA receberam $1 \times 10^{8}$ UFC de $L$. reuteri e $4 \mathrm{~g}$ de goma de guar parcialmente hidrolisada e inulina (duas vezes por dia). Além da suplementação, houve intervenção dietética, e após três meses houve redução significativa do grau de esteatose hepática, peso, índice de massa corporal, circunferência da cintura e ácido úrico; não houve alteração nas enzimas hepáticas e perfil lipídico. Diferentemente do estudo de Manzhalii et al. (2017), os participantes com EHNA, após intervenção com simbiótico composto por múltiplas cepas probióticas e frutooligossacarídeos por 12 semanas, apresentaram diminuição significativa nos níveis de ALT, AST, colesterol, fibrose hepática e IMC em comparação com o grupo controle.

A pesquisa de Asgharian et al. (2017) avaliou os efeitos da suplementação de simbióticos sobre a composição corporal e perfil lipídico em adultos DHGNA com média de IMC $29.58 \pm 0.76$ (sobrepeso/obesidade). Após a intervenção de 8 semanas o grupo simbiótico apresentou diminuição significativa do peso, gordura corporal e colesterol total. Não houve mudanças significativas no TG, HDL, glicemia em jejum, CC e IMC. Diferentemente destes autores, Mofidi et al. (2017) analisaram a suplementação de simbióticos em indivíduos com DHGNA com IMC $\leq 25 \mathrm{~kg} / \mathrm{m}^{2}$ (eutróficos) por 28 semanas. Após a intervenção o grupo simbiótico apresentou redução significativa da esteatose hepática, fibrose hepática, AST, colesterol total, glicemia em jejum, triglicerídeos e PCR, NF-KB p65. Os outros parâmetros analisados - insulina, HOMA-IR, $\mathrm{HDL}$, LDL - não apresentaram alterações significativas em comparação com o grupo placebo.

Samy e Hassanian (2011) citam que a paraoxonase antioxidante hepática 1 (PON1) é uma enzima associada à síntese de HDL principalmente no fígado, e que na doença hepática crônica níveis séricos de PON1 diminuem. A redução da atividade do PNO1 está associada ao metabolismo alterado das lipoproteínas, com a diminuição significativa HDL-colesterol e aumento dos níveis de LDL-colesterol.

Nesse contexto, Javadi et al. (2018) avaliou os efeitos da suplementação de prebióticos probióticos e simbióticos no estresse oxidativo e marcadores inflamatórios em portadores de DGHNA. O grupo que recebeu probiótico + prebiótico (simbiótico) apresentou resultados significativos na maioria dos parâmetros avaliados. Houve redução significativa na PCR, malon- dialdeído (MDA) e capacidade antioxidante total. Nenhum dos grupos apresentou, entretanto, diminuição significativa do IL-6 e TNF- $\alpha$.

De acordo Samy e Hassanian (2011), oxidação de ácidos graxos livres resulta no processo de peroxidação lipídica; produtos finais desta produzem o MDA; este apresenta propriedades pró-inflamatórias e profibrogênicas que podem agravar a lesão hepatocelular. $\mathrm{O}$ aumento dos produtos da peroxidação lipídica em pacientes com DHGNA cria e amplifica o estresse oxidativo, ocasionado o desequilíbrio entre antioxidantes e pró-oxidantes. Por sua vez, o estresse oxidativo pode causar lesões hepáticas, levando ao desenvolvimento de EHNA.

Além da suplementação em cápsulas, os probióticos e simbióticos podem ser consumidos de diversas formas, como iogurte, leites fermentados, queijos e outros alimentos fermentados (SÁEZ-LARA et al., 2016). No estudo de Bakhshimoghaddam et al. (2018) foi avaliado os benefícios de um iogurte simbiótico com Bifidobacterium animalis subsp. lactis como probiótico e inulina em portadores de DHGNA. Após 24 semanas o grupo que recebeu o iogurte simbiótico apresentou diminuição no grau da DHGNA e enzimas hepáticas (AST, AGL e ALP), aumento da CTRP-5, GLP2 , capacidade antioxidante total e sensibilidade quantitativa à insulina (QUICKI) em comparação com os outros grupos (iogurte convencional e controle). Não houve alteração significativa na insulina sérica.

Nos estudos de Famouri et al. (2017), Kobyliak et al. (2018), Duseja et al. (2019), Ferolla et al. (2016), Manzhalii et al. (2017) e Asgharian et al. (2017), além da suplementação de probióticos ou simbióticos, os participantes foram orientados a seguir um estilo de vida saudável realizando uma dieta prescrita e prática de atividade física. Segundo, Cicero, Colletti e Bellentani (2018), na ausência de tratamento farmacológico específico o principal tratamento da DHGNA, atualmente, é melhoria do estilo de vida por intermédio da alimentação e da prática de atividades físicas. Como os fatores de risco da DHGNA são semelhantes aos das doenças cardiovasculares (DCVS), as sugestões para o tratamento são as mesmas sugeridas pelas diretrizes para a prevenção das DCVS.

É importante mencionar as limitações da presente revisão. A busca restringiu-se apenas aos periódicos indexados nas bases de dados MedLine via PubMed, Web Of Science e Scopus e os estudos encontrados apresentaram heterogenicidade das amostras (tamanho, faixa etária, etnia), parâmetros avaliados e intervenções (composição dos probióticos e simbióticos utilizados e mudança no estilo de vida). 


\section{CONCLUSÃO}

A suplementação de probióticos e simbióticos tem apresentado resultados promissores na DHGNA. A maioria dos ensaios clínicos analisados demonstrou redução da concentração sérica das enzimas hepáticas (AST e ALT), melhora do perfil lipídico e diminuição do grau de esteatose hepática. Há, entretanto, ainda, poucos ensaios clínicos publicados, e os estudos foram realizados com diferentes espécies de cepas probióticas e prebióticos, dose e tempo de intervenção, portanto não há uma indicação específica para a suplementação. Sugere-se realização de mais estudos com a finalidade de esclarecer a efetividade dos probióticos e simbióticos na DHGNA.

\section{REFERÊNCIAS}

ASGHARIAN, A. et al. The effect of symbiotic supplementation on liver enzymes, c-reactive protein and ultrasound findings in patients with non-alcoholic fatty liver disease: a clinical trial. International Journal of Preventive Medicine, v. 7, 2016.

ASGHARIAN, A. et al. The effect of synbiotic supplementation on body composition and lipid profile in patients with nafld: a randomized, double blind, placebo-controlled clinical trial study. Iranian Red Crescent Medical Journal, v. 19, n. 4, 2017.

BAKHSHIMOGHADDAM, F. et al. Daily consumption of synbiotic yogurt decreases liver steatosis in patients with nonalcoholic fatty liver disease: a randomized controlled clinical trial. The Journal of nutrition, v. 148, n. 8, p. 1.276-1.284, 2018.

CAKIR, M. et al. Effects of long-term synbiotic supplementation in addition to lifestyle changes in children with obesity-related non-alcoholic fatty liver disease. Turkish Journal of Gastroenterology, v. 28, n. 5, p. 377-383, 2017.

CICERO, A. F. G.; COLLETTI, A.; BELLENTANI, S. Nutraceutical approach to non-alcoholic fatty liver disease (NAFLD): the available clinical evidence. Nutrients, v. 10, n. 9, p. 1153, 2018.

COTRIM, H. P. et al. Nonalcoholic fatty liver disease Brazilian society of hepatology consensus. Arquivos de gastroenterologia, v. 53, n. 2, p. 118-122, 2016.

DUSEJA, A. et al. High potency multistrain probiotic improves liver histology in non-alcoholic fatty liver disease (NAFLD): a randomised, double-blind, proof of concept study. BMJ Open Gastroenterology, v. 6, n. 1, 2019.

EKHLASI, G. et al. Effects of symbiotic and vitamin E supplementation on blood pressure, nitric oxide and inflammatory factors in non-alcoholic fatty liver disease. Excli Journal, v. 16, p. 278, 2017.

FAMOURI, F. et al. Effects of probiotics on nonalcoholic fatty liver disease in obese children and adolescents. Journal of Pediatric Gastroenterology and Nutrition, v. 64, n. 3, p. 413417, 2017.
FEROLLA, S. M. Microbiota intestinal e doença gordurosa hepática. Revista Brasileira de Nutrição Funcional, ed. 72, 2017.

FEROLLA, S. M. et al. Beneficial effect of synbiotic supplementation on hepatic steatosis and anthropometric parameters, but not on gut permeability in a population with nonalcoholic steatohepatitis. Nutrients, v. 8, n. 7, p. 397, 2016.

GOLDNER, D.; LAVINE, J. E. NAFLD in Children: Unique Considerations and Challenges. Gastroenterology, v. 158, n. 7, p. 1.967-1.983, 2020.

JAVADI, L. et al. Pro-and prebiotic effects on oxidative stress and inflammatory markers in non-alcoholic fatty liver disease. Asia Pacific Journal of Clinical Nutrition, v. 27, n. 5, p. 1031, 2018.

KOBYLIAK, Nazarii et al. A multi-strain probiotic reduces the fatty liver index, cytokines and aminotransferase levels in NAFLD patients: evidence from a randomized clinical trial. Journal of Gastrointestinal and Liver Diseases, v. 27, n. 1, p. 41-49, 2018.

MA, J.; ZHOU, Q.; LI, H. Gut microbiota and nonalcoholic fatty liver disease: insights on mechanisms and therapy. $\mathrm{Nu}$ trients, v. 9, n. 10, p. 1.124, 2.017.

MANTOVANI, A.; TARGHER, G.; ZOPPINI, G. Nonalcoholic Fatty Liver Disease and Implications for Older Adults with Diabetes. Clinics in Geriatric Medicine, v. 36, n. 3, p. 527547, 2020.

MANZHALII, E. et al. Treatment efficacy of a probiotic preparation for non-alcoholic steatohepatitis: a pilot trial. Journal of Digestive Diseases, v. 18, n. 12, p. 698-703, 2017.

MOFIDI, F. et al. Synbiotic supplementation in lean patients with non-alcoholic fatty liver disease: a pilot, randomised, double-blind, placebo-controlled, clinical trial. British Journal of Nutrition, v. 117, n. 5, p. 662-668, 2017.

MOHER, D. et al. Prisma statement. Epidemiology, v. 22, n. 1, p. 128, 2011.

NOBILI, V. et al. Bifidobacteria and lactobacilli in the gut microbiome of children with non-alcoholic fatty liver disease: which strains act as health players? Archives of Medical Science: AMS, v. 14, n. 1, p. 81, 2018.

ROEVER, L. Compreendendo os estudos de revisão sistemática. Revista da Sociedade Brasileira de Clínica Médica, v. 15, n. 2, p. 127-130, 2017.

SÁEZ-LARA, M. J. et al. Effects of probiotics and synbiotics on obesity, insulin resistance syndrome, type 2 diabetes and non-alcoholic fatty liver disease: a review of human clinical trials. International Journal of Molecular Sciences, v. 17, n. 6, p. 928, 2016.

SAMY, W.; HASSANIAN, M. A. Paraoxonase-1 activity, malondialdehyde and glutathione peroxidase in non-alcoholic fatty liver disease and the effect of atorvastatin. Arab Journal of Gastroenterology, v. 12, n. 2, p. 80-85, 2011.

SANTOS, C. M. C.; PIMENTA, C. A. M.; NOBRE, M. R. C. A estratégia PICO para a construção da pergunta de pesquisa e busca de evidências. Revista Latino-Americana de Enfermagem, v. 15, n. 3, p. 508-511, 2007. 
SATO, K. et al. Vitamin E has a beneficial effect on nonalcoholic fatty liver disease: a meta-analysis of randomized controlled trials. Nutrition, v. 31, n. 7-8, p. 923-930, 2015.

SCHEID, L. et al. Nutrição na doença hepática gordurosa não alcoólica e síndrome metabólica: uma revisão integrativa. Arquivos de Ciências da Saúde da UNIPAR, v. 22, n. 2, 2018.

SWEET, P. H.; KHOO, T.; NGUYEN, S. Nonalcoholic fatty liver disease. Primary Care: Clinics in Office Practice, v. 44, n. 4, p. 599-607, 2017.

TAGLIARI, E. et al. Impacto do uso de simbiótico na doença hepática gordurosa não alcoólica em modelo de ratos. Arquivos Brasileiros de Cirurgia Digestiva, São Paulo, v. 30, n. 3, p. 211-215, 2017.

VERAS Rafael dos Santos Cruz. Perfil das doenças hepáticas crônicas no ambulatório do Unifeso. Cadernos da Medicina - Unifeso, v. 1, n. 1, 2018. 